\title{
FOREWORD
}

For reprint orders, please contact: reprints@futuremedicine.com

\section{Confronting the emerging threats from zoonotic and mosquito-borne viruses}

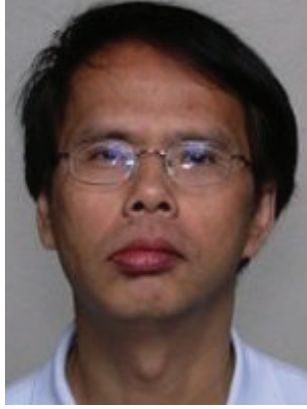

Jonathan P Wong*
"Currently, there are no approved vaccines or antiviral drugs against any of the mosquito-borne viruses, despite the fact that more than 3 billion people worldwide are at risk...”
Our world has witnessed an unprecedented number of outbreaks caused by zoonotic and mosquito-borne viruses in recent years. Viruses such as Ebola, avian influenza, SARS, dengue and others emerged or re-emerged without warning and caused widespread outbreaks with devastating effects on human lives, economy and/or international travel. Unfortunately, these viruses are often difficult to protect against because they can constantly mutate, are unpredictable and there are a critical lack of approved vaccines and antiviral drugs against them.

This themed issue entitled 'Emerging zoonotic and mosquito-borne virus diseases' brings together a collection of highly relevant and important research articles, reviews, expert perspectives, covering a number of zoonotic and mosquito-borne viral diseases. Written by a panel of leaders in the field of infectious diseases, this themed issue aims to contribute to our understanding of these often enigmatic viruses, especially relating to their pathogenesis and disease transmission. It also underscores the importance of vigilant surveillance and monitoring of disease outbreaks globally, and highlights the urgent need for more efforts and resources on novel medical countermeasure development such as antivirals drugs and vaccines needed to combat future outbreaks or pandemics. This themed issue is divided into two sections: 'emerging zoonotic' and 'emerging mosquito-borne' viruses.

\section{Emerging zoonotic viruses}

The current Ebola outbreak in West Africa perhaps best exemplifies how a largely unknown zoonotic virus can suddenly emerged without warning and caused one of the major public health crises of modern times. In the 'Ask the Experts' segment, a panel of experts consisting of a medical epidemiologist, a hemorrhagic fever infectious expert and a special pathogen medical doctor (M Borchert, A Saez and T Kratz, respectively) shared their individual and life-transforming experiences working in West Africa with the various Ebola response teams [1]. These valuable experiences and insights serve as lessons from the Ebola crisis, which can help public health, pharmaceutical and government leaders to be better prepared for future outbreaks and pandemics caused by Ebola and other emerging zoonotic viruses. Similarly, E Newman's editorial provided unique perspectives from a front-line virologist on the many challenges associated with field diagnosis, sample handling, isolation and containment of Ebola-infected patients during the West Africa outbreak [2]. D Dionisio's editorial outlined strategic and logistic lessons which can be learned from the Ebola crisis in West Africa [3]. These include equipping underdeveloped countries with knowledge, expertise

*Defence Research \& Development Canada, Suffield Research Centre, Ralston, Alberta, Canada; Tel.: +1 4035444689 ; 


\section{"Much concern about another devastating influenza pandemic similar to the Spanish flu has been triggered by the emergencies of the multiple avian influenza outbreaks in recent years."}

and resources, as well as establishing key-working partnerships among various international health agencies, government organizations and public health leaders to coordinate efforts and plans to combat the disease outbreaks.

In an interview with the Commissioning Editor for this themed issue, Emily Hargrave, L Zeitlin, President of Mapp Biopharmaceutical, Inc., and LeafBio, Inc., (USA), described an exciting breakthrough in the postexposure treatment of a limited number of Ebola patients using ZMapp ${ }^{\mathrm{TM}}$ (Mapp Biopharmaceutical, Inc., CA, USA) [4]. The development of ZMapp, a plant-expressed cocktail of monoclonal antibodies, involves the successful partnerships between US, Canadian and German scientists, as well as the US FDA and other government agencies. Among the many other important efforts to develop safe and effective antivirals to combat Ebola, J Madara's article focuses on the matrix protein of Ebola virus, a key viral protein for controlling gene transcription and viral assembly, as a promising target for antiviral drug development [5]. JS Findlay et al. eloquently characterized cytokine storm as a significant contributing factor of mortality in humans induced by zoonotic hemorrhagic fever viruses including Ebola and Marburg viruses, and explored the feasibility of mitigating hypercytokinemia as a novel treatment option to treat viral hemorrhagic fever [6]. Another zoonotic hemorrhagic fever virus endemic to the West Africa region which kills thousands of people each year is Lassa fever virus. J Hartnett $e t$ al. provides a comprehensive review on the current and emerging strategies for the diagnosis, prevention and treatment of Lassa fever [7]. This review captures the many innovative and potentially breakthrough approaches that address the unmet need for the life-saving countermeasures including antiviral drugs and vaccines needed for the prevention and treatment of Lassa fever.

Much concern about another devastating influenza pandemic similar to the Spanish flu has been triggered by the emergencies of the multiple avian influenza outbreaks in recent years. X Fan et al. outlined the complexity and challenges of vaccine strategies against the highly pathogenic avian influenza viruses in China, the epicenter for the emergence of novel strains of avian influenza and the impacts of widespread vaccination practices on public health [8]. Wong's review examined the potential role of a number of leading experimental antiviral drugs to combat pandemic influenza, and the need to expedite advanced development and regulatory approval of these drugs prior to future pandemics [9]. Additionally, A Takada's review examined the role of neutralizing monoclonal and polyclonal antibodies for use to combat future influenza pandemics and highlighted the potential and limitations of these antibodies against constantly mutating viruses [10]. M Edelstein and D Heymann provided an overview on the epidemiological and virological surveillance for the MERS-CoV infections and proposed a comprehensive strategy to limit the global spread of MERS-CoV [11]. M Aljofan provided insightful perspectives on henipaviruses, including the Hendra and Nipah viruses, which cause highly fatal encephalitic and pneumonic syndromes, to which there are no effective approved postexposure therapy in humans [12]. His article suggests that research and development of novel antiviral drugs for postexposure treatment remains a top priority to control spread and transmission from zoonotic sources to humans. Rabies is a vaccine preventable zoonotic viral disease which kills tens of thousands of people, mainly children, globally each year. C Rupprecht's insightful perspectives on rabies highlights the many valuable challenges and lessons on the prevention and treatment of rabies and suggests how simple measures such as simplifying vaccination schedule and delivery, and development of biological therapeutics, may play a significant role in lessening the impacts of rabies [13].

\section{Emerging mosquito-borne viruses}

Dengue fever, yellow fever, Chikungunya, alphavirus encephalitis and West Nile are mosquito-borne viral diseases, which are emerging or re-emerging with a vengeance in recent years. Climate change, massive deforestation and international travel are among the leading contributing factors, and these are expected to rise steadily leading to more people being at risks to be inflicted with these diseases. Currently, there are no approved vaccines or antiviral drugs against any of the mosquito-borne viruses, despite the fact that more than 3 billion people worldwide are at risk to be exposed to the dengue fever virus alone. An important strategy for reducing the transmission of dengue and other mosquito-borne diseases to humans is conventional vector control. K Olson and A Franz provided an overview of the many exciting advances and innovations in the deployment of the genetically modified mosquito Aedes aegypti to limit the spread and transmission of dengue, as well as the potential challenges in the field of implementation of these approaches [14]. Much 
progress had been made on the advanced development of the tetravalent dengue vaccine (CYDTDV) in recent years and it is the most advanced and most promising vaccine tested in Phase III trials, but questions are still raised about whether this vaccine offers adequately high efficacy to all four serotypes of dengue fever virus. D Chao and colleagues presented a unique and promising approach to enhance the efficacy of dengue vaccines by applying the principle of immunemodulation involving the use of cross-reactivity reduction immunogens [15]. It is envisaged that this approach will likely to enhance the efficacy of tetravalent or other future novel vaccines against all four serotypes of dengue fever virus. In terms of progress in antiviral drug development against dengue, V Castilla $e t$ al. highlighted the unmet need for approved antiviral drugs against dengue fever virus infection [16]. His review examined the mechanism of entry and trafficking of the dengue fever virus into host cells, and how novel antiviral drugs could be designed to block the entry and internationalization of the virus. J Wu's excellent review on the pathogenesis of the encephalitic alphaviruses revealed their interferon-interfering mechanism to evade the host's antiviral response, and how this understanding could pave the way for the development of novel interferon-inducing medical countermeasures can be used to prevent and treat infections by these interferon-evading viruses [17].

\section{Acknowledgements}

The author of this Foreword wishes to express his gratitude to all of the authors of this Special Focus issue for taking invaluable time amidst their hectic schedule to share their

\section{References}

1 Borchert M, Saez A, Kratz T. Ask the Experts: a closer look at the Ebola outbreak in West Africa. Future Virol. 10(5), 483-490 (2015).

2 Newman ENC. What are the challenges associated with testing for Ebola? A focus on the 2014 outbreak in West Africa. Future Virol. 10(5), 469-471 (2015).

3 Dionisio D. Lessons from Ebola crisis. Future Virol. 10(5), 473-47 (2015).

4 Zeitlin L. Therapeutic development and use before and during the Ebola outbreak. Future Virol. 10(5), 481-482 (2015).

5 Madara JJ, Han Z, Ruthel G, Freedman BD, Harty RN. The multi-functional Ebola virus VP40 matrix protein is a promising

insights and knowledge, and in so doing, contributed greatly to both the scientific and the medical communities in our collective fight against these viruses. The author also wishes to express his sincere gratitude to Emily Hargrave, the Commissioning Editor of Future Virology, for her great vision and leadership in bringing this important themed issue into fruition. We also acknowledge members of the editorial board for Future Virology, for their outstanding collaboration and assistance. We would like to dedicate this themed issue to more than 250 frontline Ebola workers, including physicians, nurses and healthcare workers who gave up their lives while unselfishly serving and treating Ebola patients during the crisis. Their passion for their work and love for the patients are a shining light to all and their heroism can never be forgotten.

\section{Financial \& competing interests disclosure}

The author has no relevant affliation or financial involvement with any organization or entity with a financial interest in or financial conflict with the subject matter or materials discussed in this manuscript. This includes employment, consultancies, honoraria, stock ownership or options, expert testimony, grants or patents received or pending or royalties.

No writing assistance was utilized in the production of this manuscript.

\section{Open access}

This work is licensed under Crown copyright protection and licensed for use under the Open Government Licence unless otherwise indicated. Where any of the Crown copyright information in this work is republished or copied to others, the source of the material must be identified and the copyright status under the Open Government Licence acknowledged.

therapeutic target. Future Virol. 10(5), 537-546 (2015).

6 Findlay JS, Ulaeto D, D'Elia RV. Cytokines and viral hemorrhagic fever: potential for therapeutic intervention. Future Virol. 10(5), 547-557 (2015).

7 Hartnett JN, Boisen ML, Oottamasathien D et al. Current and emerging strategies for the diagnosis, prevention and treatment of Lassa fever. Future Virol. 10(5), 559-584 (2015).

8 Fan X, Hu Y, Zhang G, Wang M. Veterinary influenza vaccines against avian influenza in China. Future Virol. 10(5), 585-595 (2015).

9 Wong JP. Experimental antiviral drugs against pandemic influenza. Future Virol. 10(5), 597-607 (2015).
10 Takada A. The clinical potential of passive immunization with therapeutic antibodies: focus on highly pathogenic avian influenza virus infection. Future Virol. 10(5), 491-496 (2015).

11 Edelstein M, Heymann DL. What needs to be done to control the spread of MERS-CoV? Future Virol. 10(5), 497-505 (2015).

12 Aljofan M. Henipaviruses: are not yet history. Future Virol. 10(5), 507-515 (2015).

13 Rupprecht CE, Kuzman IV. Why we can prevent, control and possibly treat - but will not eradicate - rabies. Future Virol. 10(5), 517-535 (2015).

14 Olson KE, Franz AWE. Advances in genetically modified Aedes aegypti to 
control transmission of dengue viruses. Future Virol. 10 (5), 609-624 (2015).

15 Chao D-Y, Crill WD, Davis BS, Chang G-JJ. Can reductions in the cross-reactivity of flavivirus structural proteins lead to improved safety and immunogenicity of tetravalent dengue vaccine development? Future Virol. 10 (5), 477-480 (2015).

16 Castilla V, Piccini LE, Damonte EB. Dengue virus entry and trafficking: perspectives as antiviral target for prevention and therapy. Future Virol. 10(5), 625-645 (2015).
$17 \mathrm{Wu} \mathrm{JQH}$. Virulence determinants of New World alphaviruses and broad-acting therapeutic strategies. Future Virol. 10(5), 647-657 (2015). 